\title{
Erratum to: Shared quantum control via sharing operation on remote single qutrit
}

\author{
Daochu Liu • Yimin Liu • Chuanmei Xie • \\ Xiaofeng Yin · Xiansong Liu · Zhanjun Zhang
}

Published online: 24 August 2013

(C) Springer Science+Business Media New York 2013

\section{Erratum to: Quantum Inf Process DOI 10.1007/s11128-013-0615-8}

There is an error in the original version of this article. Specifically, the operators $W$ 's defined by the Eq. (20) in the original version should be unitary in physics. This requirement directly leads to a constraint $x x^{*}=y y^{*}=z z^{*}=1 / 3$. Unfortunately, the constraint is neglected in the original version. By virtue of the constraint, the three cases (1-3) following the Eq. (20) in the original version should be removed, for the corresponding operators $W$ 's there are non-unitary. Nonetheless, the unitarity of the operators $W$ 's in the case (4) is satisfied, and hence, the case (4) can be kept. In this condition, one can easily find that an arbitrary operation $U$ can only be shared with probability $1 / 3$. However, if it is known to be diagonal, i.e., belong to the set $U^{(1)}$ in the original version, then the sharing of it can be achieved in a deterministic manner. In terms of the above descriptions, all the irrelative statements in the discussion in the original version should be simply removed.

The online version of the original article can be found under doi:10.1007/s11128-013-0615-8.

D. Liu $\cdot$ C. Xie $\cdot$ X. Yin $\cdot$ X. Liu $\cdot$ Z. Zhang $(\varangle)$

School of Physics and Material Science, Anhui University,

Hefei 230039, Anhui, China

e-mail: zjzhang@ahu.edu.cn

Y. Liu

Department of Physics, Shaoguan University,

Shaoguan 512005, China 